\title{
Determinação de parâmetros farmacognósticos para as folhas de Erythroxylum suberosum A. St.-Hilaire (Erythroxylaceae) coletadas no município de Goiânia, GO.
}

RODRIGUES, G.A.'; SOUZA, W.C.'; GODINHO, M.G.C.'; FERREIRA, H.D.2; VILA VERDE, G.M.1* 'Universidade Estadual de Goiás, Caixa Postal 459, 75000-000 Anápolis-GO, Brasil. 2Universidade Federal de Goiás, Campus Samambaia, Caixa Postal 131,74001-970 Goiânia-GO, Brasil. *Autor para correspondência: giuliana.muniz@ueg.br

RESUMO: As plantas medicinais representam um dos principais recursos terapêuticos utilizado pelo homem para a cura e prevenção de doenças, no entanto, para que o tratamento seja seguro e eficaz é necessário conhecer seus componentes químicos. Dessa forma, o intuito deste trabalho foi determinar os parâmetros farmacognosticos para o estabelecimento do controle de qualidade da droga vegetal composta pelas folhas de Erythroxylum suberosum A. St.-Hil., pertencentes à família Erythroxylaceae ocorrente no Cerrado. Para tanto, foram coletadas folhas adultas nas proximidades da Universidade Federal de Goiás, GoiâniaGO. Foram determinados o teor de umidade por meio de balança com irradiação de raios infravermelho e o teor de cinzas totais. Na prospecção fitoquímica foram pesquisadas as classes de metabólitos secundários e realizou-se o doseamento de fenóis totais, flavonoides e taninos totais. Os resultados dos testes de pureza realizados, teor de umidade e teor de cinzas totais, estão de acordo com os limites estabelecidos pelas especificações farmacopeicas. As análises fitoquímicas evidenciaram a presença de flavonoides, taninos, cumarinas, saponinas e resinas. No doseamento de fenóis totais, taninos e flavonoides presentes nas folhas de $E$. suberosum A. St.-Hil. obteve-se respectivamente 17,97\%, 6,31\%, 3,87\%. Estes resultados confirmam os dados da literatura quanto à presença destes compostos em Erythroxylaceae, pois de acordo com nas folhas de E. tortuosum obteve-se valores de 10\%, 8,4\% e 0,064\% de Fenóis, Taninos e flavonoides, respectivamente e nas folhas de $E$. deciduum foram encontrados $12,04 \%$ de fenóis totais, $0,87 \%$ de taninos e $1,37 \%$ de flavonoides. Os resultados encontrados no presente trabalho também se tornam relevantes quando comparadas as quantidades desses metabólitos com espécies clássicas na biossíntese de compostos fenólicos como o teor de taninos de Stryphnodendron adstringens (Mart.) Coville e em Eugenia uniflora L. foi de $29,9 \%$ e 2,96\%, respectivamente. Teores de flavonóides que variaram entre $0,65 \%$ a $0,79 \%$ na Calendula officinalis L. e Ginkgo biloba L. 0,59\%, 0,75\% e 0,79\%. Sendo assim, a quantidade considerável de compostos fenólicos obtida em E. suberosum A. St.-Hil. sugere que a espécie possua um importante potencial terapêutico e quem sabe potencial antioxidante, a ser explorado em estudos posteriores.

Palavras-chave: Plantas medicinais. Prospecção fitoquímica. Fenois. Erythroxylum suberosum A. St.-Hil.

\begin{abstract}
Determination of pharmacognostic parameters for the leaves of Erythroxylum suberosum A. St.-Hilaire (Erythroxylaceae) collected in the city of Goiania, GO. Medicinal plants represent a major therapeutic resources used by man for the cure and prevention of diseases, however, that the treatment be safe and effective it is necessary to know their chemical components. Thus, the aim of this work was to determine the pharmacognostic parameters for the establishment of the plant drug quality control made by the leaves of Erythroxylum suberosum A. St.-Hil., belonging to the family Erythroxylaceae occurring in the Cerrado. To this end, adult leaves were collected near the Federal University of Goiás, Goiânia-GO. It was determined the moisture content through scale radiating infrared rays and the content of total ash. The phytochemical screening of secondary metabolites classes were surveyed and performed the determination of total phenols, flavonoids and total tannins. The results of the purity tests, moisture content and total ash content, are in accordance with the limits established by the
\end{abstract}

Recebido para publicação em 09/09/2014

Aceito para publicação em 21/01/2015

10.1590/1983-084X/14_114

Rev. Bras. Pl. Med., Campinas, v.17, n.4, supl. III, p.1169-1176, 2015. 
pharmacopeic specifications. The phytochemical analysis revealed the presence of flavonoids, tannins, coumarins, saponins and resins. The determination of total phenols, tannins and flavonoids quantities present in the leaves of $E$. suberosum A. St.-Hil. were obtained respectively as $17.97 \%, 6.31 \%, 3.87 \%$. These results confirm the literature data for the presence of these compounds in Erythroxylaceae, since according to the leaves of $E$. tortuosum yielded values of $10 \%, 8.4 \%$ and $0.064 \%$ of phenols, tannins and flavonoids, respectively. On the E. deciduum leaves were found $12.04 \%$ of total phenols, tannins $0.87 \%$ and $1.37 \%$ of flavonoids. The results of this work also become relevant when comparing the amounts of these metabolites with classic species in the biosynthesis of phenolic compounds such as tannins content in barbatimão and pitanga was $29.9 \%$ and $2.96 \%$, respectively. Flavonoid contents ranging from $0.65 \%$ to $0.79 \%$ in calendula and ginkgo $0.59 \%, 0.75 \%$ and $0.79 \%$. Thus, considerable amount of phenolic compounds obtained in E. suberosum. A. St. Hil. It suggests that the species has an important therapeutic potential and who knows antioxidant potential to be explored in further studies.

Keywords: Medicinal plants, Phytochemical screening, phenolic compounds, Erythroxylum suberosum A. St.-Hil.

\section{INTRODUÇÃO}

As plantas medicinais compreendem um dos mais antigos recursos terapêuticos na busca de melhores condições de vida ao homem como o tratamento, cura e prevenção de doenças (Brasil, 2006). Assim, constituíram ferramentas importantes utilizadas no combate às enfermidades humanas e de animais.

Nesse sentido, a Organização Mundial da Saúde salienta a grande necessidade por parte da população dos países em desenvolvimento quanto à medicina tradicional no que concerne a sua atenção primária, tendo em vista que $80 \%$ desta população utiliza práticas tradicionais nos seus cuidados básicos à saúde e utilizam plantas ou preparações a partir destas (Brasil, 2006).

Devido as suas variedades vegetais, - Brasil é proprietário da maior biodiversidade vegetal do planeta, possuindo $20 \%$ do número total de espécies do mundo. Detendo ainda, biomas com incontáveis espécies que até o presente momento não tiveram seu potencial para exploração inteiramente esclarecido, sendo ainda que estas são de grande relevância para o estudo e descobertas de novos medicamentos (Sibio \& Rossi, 2007).

Dentre os biomas brasileiros, o Cerrado se destaca, sendo considerado o segundo maior bioma brasileiro e representa $22 \%$ do território nacional, figurando como a maior savana do mundo (Brasil, 2011). Possui uma exuberante fauna e flora, com mais de 11.000 espécies vegetais das quais 4.400 são endêmicas. Como parte da flora deste bioma encontra-se a Erythroxylaceae que é uma das famílias mais representativas do bioma, tem seus principais centros de diversidade e endemismo na América do Sul, no sudeste asiático e em Madagascar (Lorenzi, 2005).

Esta família possui quatro gêneros e aproximadamente 250 espécies (Amaral Júnior,
1980; Ribeiro et al., 1999), sendo que a maioria delas pertence ao gênero Erythroxylum P. Browne. Esse gênero é bastante distribuído nas regiões tropicais e subtropicais do mundo, sendo que no Brasil é descrito a ocorrência de cerca de 130 destas. Portanto, é considerado um dos gêneros mais comuns da vegetação do cerrado brasileiro (Souza \& Lorenzi, 2012).

Estudos realizados sobre a composição química desse gênero indicam a ocorrência de alcalóides tropânicos em folhas e frutos (El-Imam et al.,1988; Christen et al., 1995; Brachet et al., 1997; Bringmanm et al., 2000; Griffin \& Lin, 2000; Payol-Hill et al., 2000; Zuanazzi et al., 2001). Além disso, flavonóides e óleos voláteis com compostos triterpênicos têm sido relatados (Bohm et al., 1988; Kolodziej et al., 1991; Chaves et al., 1996; Barreiros et al., 2002; Johnson et al., 2003; Catarino et al., 2007).

A partir do estudo fitoquímico realizado com diferentes espécies do gênero Erythroxylum foram isolados vários metabólitos secundários como flavonóides e alcalóides, bem como taninos, terpenos e fenilpropanóides que apresentam atividades antioxidantes, anticancerígenas, atividade anti-inflamatória dentre outras (Ansel et al., 1993, 2003). Diversas espécies desse gênero possuem propriedades medicinais, como a popular coca $(E$. coca Lamk. e E. novogranatense) e suas variedades as mais conhecidas e estudadas devido à presença de alcalóides em suas folhas, onde a cocaína pode chegar até $2 \%$ da folha de massa seca que foi empregada como anestésico local em pequenas cirurgias (Bohm et al.,1982; Plowman \& Berry, 1999; Griffin \& Lin, 2000; Bieras \& Sajo, 2004).

$\mathrm{Na}$ região do Alto Rio Grande- MG, a Erythroxylum campestre e Erythroxylum tortuosum, em termos de etnofarmacobotânica tem sido muito 
utilizadas como plantas medicinais, uma vez que o preparo de infusão das raízes de $E$. campestre e da casca do caule da E. tortuosum são administrados, respectivamente, como laxante e adstringente no caso de hemorragias (Paula, 2012).

A espécie Erythroxylum suberosum A. St.-Hil. (Figura 1) é bastante distribuída no Brasil, sendo encontrada em vinte das vinte e sete unidades federativas do Brasil e em outros países como Bolívia, Paraguai, Guiana Francesa e Venezuela (Barbosa, 2001). É conhecida popularmente como galinha-choca, mercúrio-do-campo, sessenta-edois, azougue-do-campo, cabelo-de-negro. Por ser típica do cerrado rebrota vigorosamente após as queimadas. Floresce de agosto a dezembro e frutifica de setembro a janeiro (Mendonça et al., 1998).

Segundo Barbosa (2001), a E. suberosum compreende um subarbusto a arvoreta de até 6 metros de altura, densamente ramificado com ramos cilíndricos, grossos, ramos novos comprimidos, castanhos, córtex castanho acinzentado, bastante suberoso nos velhos, com fissuras e esfoliativo como mostra a Figura 2.

A referida espécie tem utilização limitada pela espessura e rigidez da madeira vermelhoescura, não permitindo seu uso na marcenaria e carpintaria. A casca suberosa adstringente é útil

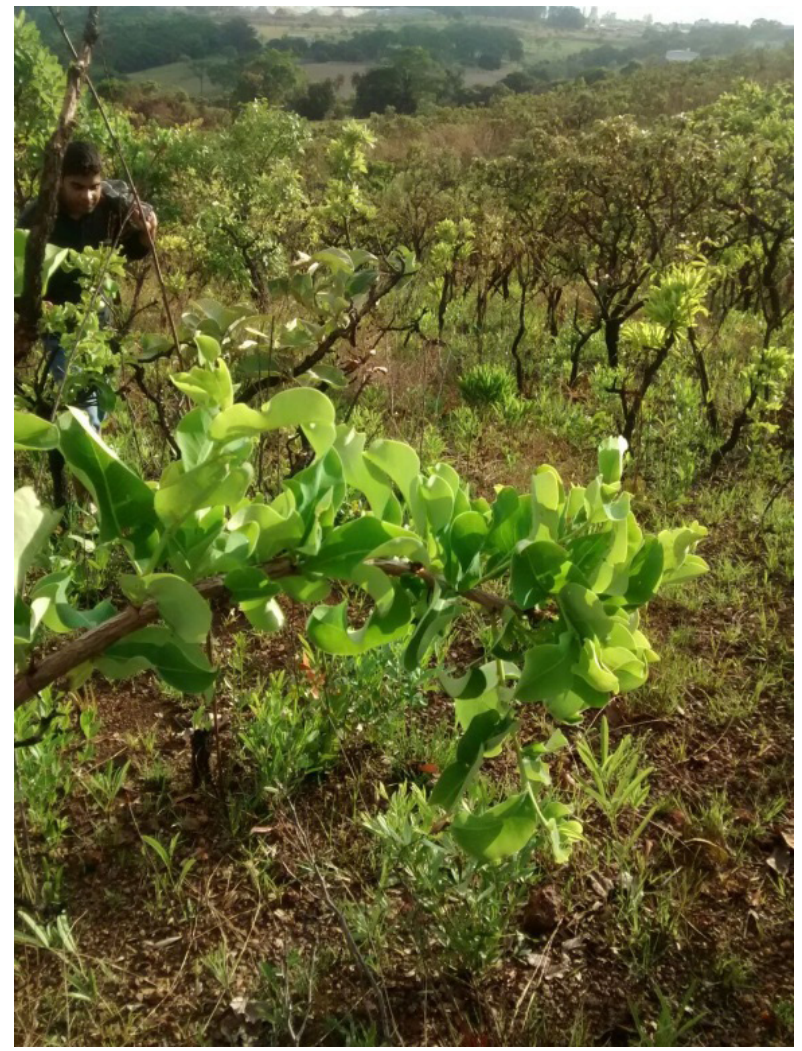

FIGURA 1. Indivíduo Erythroxylum suberosum A. St.-Hil.

Fonte: Moreira e Santos (2015)

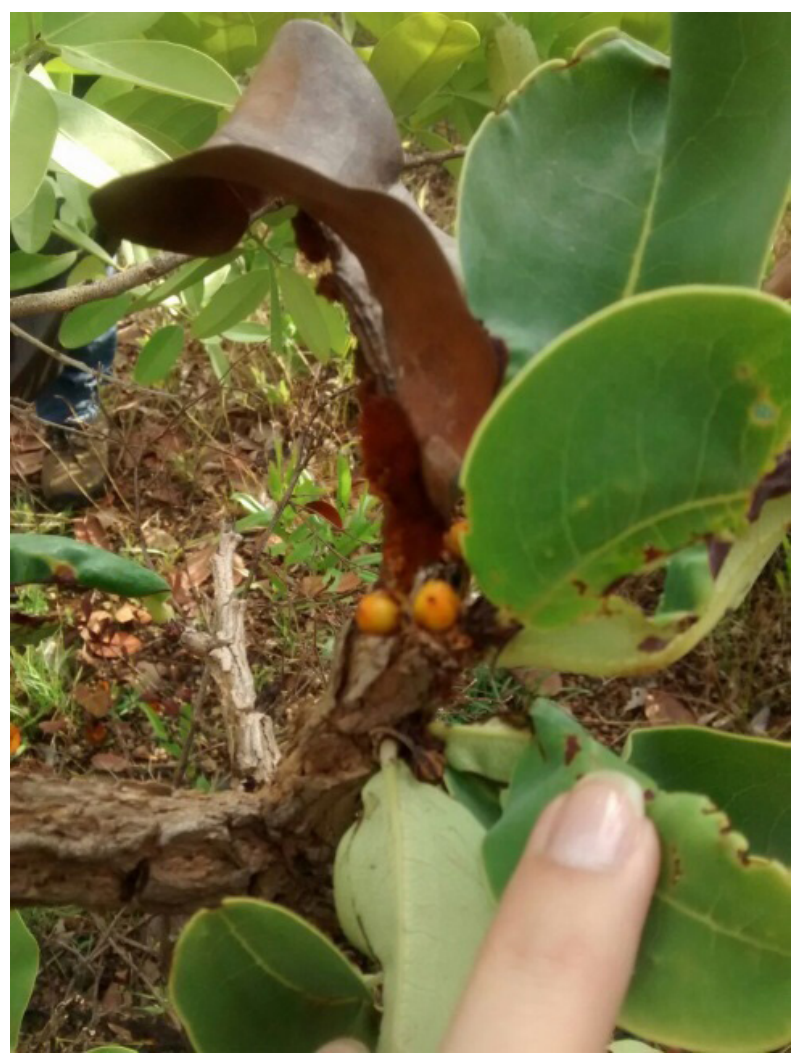

FIGURA 2. Ramo com caule suberoso e frutos laranja-avermelhados.

Fonte: Moreira e Santos (2015)

para curtume e também fornece matéria tintorial castanho-avermelhada, que dizem ter fixidez. Seus frutos são dados às galinhas, cujo choco pretendese evitar (Mendonça et al., 1998). Entretanto, a espécie ainda carece de estudos farmacognósticos e farmacológicos para comprovação de seu poder medicinal como a adstringência, por exemplo, evidenciada no conhecimento popular. Assim, este trabalho objetiva identificar as classes de metabólitos secundários e o doseamento de fenóis totais, flavonóides totais e taninos totais presentes nas folhas de E. suberosum A. St.-Hil., ocorrente em "senso stricto" no município de Goiânia-GO, bem como determinar o teor de umidade e de cinzas totais que são parâmetros importantes para o controle de qualidade da droga vegetal.

\section{MATERIAL E MÉTODO \\ Material vegetal}

Para a realização deste estudo foram utilizadas folhas adultas e sadias de $E$. suberosum coletadas nas proximidades da Universidade Federal de Goiás, Goiânia-GO, nas posições geográficas $16^{\circ} 33^{\prime} 58^{\prime \prime}$ de latitude sul e $49^{\circ} 17^{\prime}$ 11" de longitude oeste. A identificação botânica do material foi realizada pelo Prof. Dr. Heleno

Rev. Bras. PI. Med., Campinas, v.17, n.4, supl. III, p.1169-1176, 2015. 
Dias Ferreira ICB/UFG. Parte do material vegetal foi destinada à constituição da exsicata que foi depositada no Herbário da Universidade Estadual de Goiás, sob o tombo de número 5997. Após a coleta, as folhas, foram dessecadas em estufa com circulação forçada de ar a $40^{\circ} \mathrm{C}$, por 48 horas e, posteriormente submetidas à moagem em moinho de facas, modelo Marconi MA630, em granulometria adequada às análises.

\section{Determinação do teor de umidade e teor de cinzas totais}

Para a determinação da umidade através de irradiação de raios infravermelhos foi utilizado o método proposto por Amoedo \& Muradian (2002). A determinação do teor de cinzas totais procedeuse de acordo com as técnicas descritas por Costa (1982) e Pregnolatto \& Pregnolatto (1985). As análises foram realizadas em triplicata.

\section{Prospecção fitoquímica}

A análise fitoquímica do pó das folhas foi realizada em triplicata para determinação das principais classes de metabólitos secundários, conforme as técnicas descritas por Costa (1982), Matos (1988), Matos \& Matos (1989).

Para a prospecção fitoquímica foram utilizados aproximadamente $60 \mathrm{~g}$ do pó da planta e reagentes específicos, para identificação de alcalóides, esteróides e triterpenóides, heterosídeos flavonóides, taninos, cumarinas, heterosídeos saponínicos, heterosídeos antraquinônicos e resinas.

\section{Doseamento de fenóis totais, taninos totais e flavonóides totais}

Para o doseamento de fenóis totais e taninos totais presentes nas folhas de $E$. suberosum foi utilizado o método Hargerman \& Butler (1978) descrito por Waterman \& Mole (1994) e o método de Rolim et al. (2005) modificado foi utilizado para o doseamento de flavonoides totais.

Para a dosagem de fenóis totais o método baseia-se na reação de complexação dos compostos fenólicos presentes na amostra com uma solução de $\mathrm{FeCl}_{3}\left(162 \times 10^{-2} \mathrm{~g} / \mathrm{mL}\right)$ e utilizou-se o ácido tânico como solução padrão. Para o doseamento de taninos a técnica baseia-se na propriedade dos taninos de precipitar em solução aquosa na presença de proteína. Para essa técnica, utilizou-se uma solução de $1 \mathrm{mg} / \mathrm{mL}$ de albumina sérica bovina em solução tampão de acetato de sódio $0,2 \mathrm{M}(\mathrm{pH} 4,9)$ contendo $0,17 \mathrm{M}$ de cloreto de sódio para precipitar os taninos em solução. Utilizou-se a solução detergente de LSS/ Trietanolamina para separar os taninos da proteína no precipitado, e a solução de $\mathrm{FeCl}_{3}$ foi utilizada como solução cromogênica. Além disso, o ácido tânico foi utilizado como solução padrão. Para o doseamento de flavonóides totais o método baseia-se na propriedade dos mesmos em absorver radiação no comprimento de onda da luz ultravioleta (UV) proporcionalmente à sua concentração. Nesse teste utilizou-se a rutina $(0,1 \mathrm{mg} / \mathrm{mL})$ como solução padrão. A leitura da absorbância das amostras foi realizada em espectrofotômetro modelo UV-VIS Lambda 25 da Perkin Elmer, em um comprimento de onda de $510 \mathrm{~nm}$ e com os dados obtidos foram construídas as curvas de calibração padrão (Absorbância x Concentração).

Os valores das absorbâncias obtidos com as leituras das amostras foram substituídos na variável $y$, da equação $y=a+b x$, sendo que esta foi calculada através da curva padrão fornecida pelo programa Excel, obtendo-se a concentração (variável $x$ ) em mg.mL-1. Para calcular a porcentagem de fenóis totais e taninos totais presentes na amostra analisada utilizaram-se as seguintes fórmulas:

$C=\frac{\text { Absorbância-A }}{B}$

$\%$ Fenóis/taninos totais $=c \times \frac{250}{(\mathrm{~m}(\mathrm{~g})} \times 10^{-3} \times 100$

Onde: $\mathrm{c}=$ concentração de ácido tânico em $\mathrm{mg} / \mathrm{mL} ; A=$ coeficiente linear da equação da reta; $B$ = coeficiente angular da equação da reta; $\mathrm{m}=$ massa inicial descontado o teor de umidade na amostra

Para calcular a porcentagem de flavonoides presentes na amostra foram utilizadas as seguintes fórmulas:

$$
\begin{gathered}
C=\frac{\text { Absorbância-A }}{B} \\
\text { \%Fenóis/taninos totais }=c \times \frac{500}{(\mathrm{~m} \mathrm{(g)}} \times 10^{-3} \times 100
\end{gathered}
$$

Onde: $\mathrm{c}=$ concentração de rutina em $\mathrm{mg} /$ $\mathrm{mL} ; \mathrm{A}=$ coeficiente linear da equação da reta; $\mathrm{B}=$ coeficiente angular da equação da reta; $m=$ massa inicial descontado o teor de umidade na amostra

\section{RESULTADOS E DISCUSSÃO}

Os teores de umidade e cinzas totais analisados nas folhas de $E$. suberosum estão apresentados na Tabela 1 abaixo. As principais classes de metabólitos secundários encontrados na prospecção fitoquímica estão demonstradas na Tabela 2. Os resultados encontrados no doseamento de fenóis totais, taninos e flavonoides estão descritos na Tabela 3.

As matérias-primas vegetais contêm 
TABELA 1. Teores de umidade e teores de cinzas totais expressos em porcentagem $(p / p) \pm$ desvio padrão das amostras de folhas de $E$. suberosum A. St.-Hil.

\begin{tabular}{ccc}
\hline $\begin{array}{c}\text { Amostra } \\
\text { (triplicata) }\end{array}$ & $\begin{array}{c}\text { Teor de } \\
\text { umidade }\end{array}$ & $\begin{array}{c}\text { Teor de cinzas } \\
\text { totais }\end{array}$ \\
\hline 1 & $9 \pm 0,33$ & $1,41 \pm 0,05$ \\
2 & $8,33 \pm 0,33$ & $1,41 \pm 0,05$ \\
3 & $8,66 \pm 0,33$ & $1,42 \pm 0,05$ \\
\hline
\end{tabular}

TABELA 2. Classes de metabólitos secundários pesquisados nas amostras de folhas de $E$. suberosum A. St.-Hil.

\begin{tabular}{ll}
\hline Classes de metabólitos secundários & Resultados \\
\hline Alcalóides & - \\
Heterosídeos Cardioativos & - \\
Flavonóides & + \\
Taninos & + \\
Cumarinas & + \\
Saponinas & + \\
Antraquinonas & - \\
Resinas & + \\
\hline
\end{tabular}

(+) Presença (-) Ausência

naturalmente, certa quantidade de água, que se mantém após a secagem das plantas. É relevante conhecer este valor, pois este índice está relacionado com a correta preparação e garantia da conservação das drogas vegetais (Oliveira et al., 2005).

AE. suberosum não apresenta monografia descrita em nenhuma edição da Farmacopéia Brasileira. Dessa forma, os valores obtidos nos testes de pureza, são comparativos aos descritos nas monografias da maioria das espécies vegetais.

As matérias-primas vegetais armazenam determinada quantidade de água mesmo após serem submetidas ao processo de secagem. Dessa forma, é relevante o conhecimento deste valor para serem evitadas contaminações com micro-organismos e degradações por enzimas (Costa, 2001). O método de secagem adotado no desenvolvimento deste trabalho propiciou uma faixa de umidade para as folhas de $E$. suberosum $(8,66 \%)$ dentro do limite estabelecido pela Farmacopéia Brasileira $5^{\circ}$ ed. (2010), cujo limite máximo é de 8 a $14 \%$, o que propiciará uma boa conservação da droga vegetal. A determinação dos teores de cinzas nas amostras processadas é um teste de pureza importante porque pode permitir a identificação do produto vegetal. Fármacos vegetais, geralmente são falsificados pela adição de produtos de origem mineral em suas formulações (Cunha, 2005). Costa (2001) relata que existem cinzas fisiológicas, providas dos próprios minerais que integram as plantas e as cinzas não-fisiológicas, que são impurezas externas inorgânicas. Assim, quando o teor está acima do estabelecido para a maioria das espécies vegetais, indica que a droga vegetal está contaminada (Farias, 2004). De acordo com a Farmacopeia Brasileira (2010), os valores de cinzas totais geralmente não ultrapassam 12\%. Diante disso, pode-se inferir que as amostras de folhas de E. suberosum. não apresentam contaminação por cinzas, pois apresentaram teor de cinzas da ordem de $1,41 \%$.

Realizou-se a prospecção fitoquímica a fim de se conhecer o perfil químico de $E$. suberosum ocorrente em Goiânia-GO. A formação de espuma no teste de saponina caracterizou o teste como positivo para heterosídeos saponínicos. $\mathrm{O}$ aparecimento de coloração vermelha, presença de fluorescência e coloração amarela para reação de Shinoda, Oxalobórica e reação com hidróxidos, respectivamente, evidenciou teste positivo para flavonóides. No teste para taninos houve formação de precipitado, após adição de solução aquosa de $\mathrm{FeCl}_{3}$ e presença da coloração enegrecida caracterizando teste positivo. A observação de fluorescência, sob luz UV.,confirmou a presença de cumarinas no ensaio realizado. As resinas foram evidenciadas pela presença de turvação. Espécies de Erythroxylaceae possuem indicadores da presença de alcalóides, entretanto, houve ausência nesta amostra. Tal fato pode ser devido a diversos fatores, dentre eles, sazonalidade, local de coleta, tipo de solo, até mesmo implicações genéticas.

Segundo Oliveira et al. (2010) os alcalóides compreendem $20 \%$ dos compostos naturais descritos no gênero Erythroxylum. Dentre estes, os alcalóides tropânicos têm sido utilizados como analgésico, anti-hipertensivo, anticolinérgico, dentre

TABELA 3. Doseamentos de fenóis totais, taninos e flavonoides de amostras de folhas de Erythroxylum suberosum A. St.-Hil. expressos em porcentagem $(\mathrm{p} / \mathrm{p}) \pm$ desvio padrão.

\begin{tabular}{lllc}
\hline Amostra (triplicata) & Fenóis & Taninos & Flavonóides \\
\hline 1 & $16,97 \pm 1,97$ & $6,47 \pm 0,25$ & $3,91 \pm 0,05$ \\
2 & $16,69 \pm 1,97$ & $6,46 \pm 0,25$ & $3,89 \pm 0,05$ \\
3 & $20,24 \pm 1,97$ & $6,02 \pm 0,25$ & $3,81 \pm 0,05$ \\
\hline
\end{tabular}

Rev. Bras. Pl. Med., Campinas, v.17, n.4, supl. III, p.1169-1176, 2015. 
outros.

Um estudo desenvolvido por Bieri et al. (2006), avaliou o teor do alcalóide tropânico cocaína presente nas folhas de 51 espécies de plantas pertencentes ao gênero Erythroxylum. A cocaína foi detectada em 23 das 51 espécies examinadas, sendo que Erythroxylum suberosum demonstrou ausência total de cocaína na espécie.

No entanto, em estudo desenvolvido por Brock et al. (2005), calisteginas foram identificadas e quantificadas no gênero Erythroxylum, a maior parte das espécies analisadas continha pelo menos um dos quatro diferentes tipos de calistegina, apenas nove espécies não mostraram qualquer calistegina ou continham vestígios que não puderam ser quantificados. Foram examinadas 45 espécies pertencentes ao gênero Erythroxylum, sendo que 38 espécies apresentaram um ou mais tipos de calisteginas. A espécie $E$. suberosum coletada em Goiás apresentou três tipos de calisteginas na concentração de 200-1000 ug por grama de massa seca.

A identificação desses metabólitos nas plantas é indispensável, tendo em vista que na medicina popular, plantas ricas em taninos são empregadas no tratamento de diversas moléstias como diarreia, hipertensão arterial, hemorragias, feridas, queimaduras, reumatismo, problemas estomacais, renais, do sistema urinário e processos inflamatórios, além de serem utilizados pela indústria no curtimento de couro, na produção de borrachas, na produção de vinho e como antioxidantes (Santos \& Mello, 2004; Cunha, 2005). Os flavonoides como compostos fenólicos nas plantas exercem a proteção contra raios ultravioleta, proteção contra insetos, vírus e bactérias, atração de polinizadores, ação antioxidante, alelopática e inibição de enzimas (Zuanazzi \& Montanha, 2004).

Trabalho desenvolvido por Nascimento et al. (2012) relatou a ocorrência de cinco diterpenos com esqueleto do tipo Abietano e ent-kaurano no gênero Erythroxylum. Os cinco novos diterpenos abietano e ent-kaur-16-eno que foram isolados a partir da partição do extrato etanólico dos ramos de E. suberosum. podem ser considerados como potenciais agentes citotóxicos merecendo mais estudos biológicos. O isolamento dos glicosídeos flavonóides ombuin-3-rutinosídeo e rutina (quercetina-3-rutinosídeo) reforçaram a utilidade de flavonóides como marcadores quimiotaxonômicos para o gênero.

Os flavonoides constituem uma importante classe de polifenóis relativamente abundantes e diversificados entre os metabólitos secundários dos vegetais. Há relatos de atividade antitumoral, antiespasmódica, anti-inflamatória, antimicrobiana, antimutagênica, anti-úlcera, antiviral, antioxidante, estrogênica, giardicida, tripanossomicida, inibidor da enzima acetilcolinesterase e inibidor da enzima conversora de angiotensina, atribuídas a flavonoides. Além disso, ainda são importantes como marcadores taxonômicos devido à sua relativa abundância em quase todo o reino vegetal e sua especificidade em algumas espécies (Zuanazzi, 2001).

Tendo em vista os resultados que foram encontrados na prospecção, procedeu-se a quantificação dos metabólitos com maior potencial na medicina popular e os valores obtidos foram satisfatórios do ponto de vista terapêutico para esta espécie.

O teor de fenóis totais presentes nas folhas de $E$. suberosum apresentou um valor considerável com uma média de $17,97 \%$. Com relação aos compostos fenólicos analisados observou-se que os taninos $(6,31 \%)$ foram predominantes aos flavonóides $(3,87 \%)$. Estes resultados confirmam os dados da literatura quanto à presença destes compostos em Erythroxylaceae. De acordo com Arruda et al. (2010) nas folhas de E. tortuosum Martius obteve-se valores de 100, 84 e 0,64 mg/g da amostra de fenóis, taninos e flavonóides, respectivamente. Oliveira et al. (2014) encontrou $12,04 \%$ de fenóis totais, $0,87 \%$ de flavonóides (Metodologia descrita na Farmacopéia Brasileira $5^{\circ}$ ed. (2010) na monografia da planta Calêndula (Calendula officinalis L.) e 1,37\% de flavonóides (Método espectrofotométrico descrito por Rolim et al., 2005) nas folhas de E. deciduum A. St.-Hil.

De modo geral, a quantidade considerável de compostos fenólicos obtida em $E$. suberosum A. St.-Hil. contribui para corroborar a utilização dessa espécie na medicina popular. Isso se torna relevante quando comparada a quantidade desses metabólitos com espécies clássicas por biossintetizar compostos fenólicos. O teor de taninos doseado por Ardisson et al. (2002) no Stryphnodendron adstringens (Mart.) Coville (Barbatimão) e por Auricchio et al. (2007) na Eugenia uniflora L. (Pitanga) foi de 29,9\% e 2,96\%, respectivamente. Borella et al. (2011) encontraram teores de flavonóides que variaram entre 0,65\% a $0,79 \%$ na Calendula officinalis L. e Rostirolla et al. (2010) evidenciaram no Ginkgo biloba L. 0,59\%, 0,75\% e 0,79\% de flavonóides.

\section{CONCLUSÃO}

Os testes de pureza realizados nas partes aéreas de $E$. suberosum demonstraram que o método de secagem adotado propiciou atingir uma faixa de umidade dentro do limite estabelecido pela Farmacopéia Brasileira $5^{\circ}$ ed. (2010), bem como o teor de cinzas totais, que contribuíram para a boa conservação da droga vegetal. Os testes fitoquímicos executados indicaram a presença

Rev. Bras. PI. Med., Campinas, v.17, n.4, supl. III, p.1169-1176, 2015. 
das principais classes de metabólitos secundários: flavonóides, taninos, cumarinas, saponinas e resinas. As análises quantitativas de fenóis totais, taninos totais e flavonóides totais demonstraram a presença de fenóis em quantidades significativas, os quais podem ser responsáveis por propriedades antiinflamatórias e antioxidantes a serem investigadas e exploradas não só pela indústria farmacêutica, mas também pela indústria alimentícia e de cosméticos.

Dessa forma, a pesquisa realizada foi satisfatória para o conhecimento e caracterização da espécie $E$. suberosum, já que traz uma contribuição relevante para essa importante família botânica do Cerrado cujos estudos farmacognósticos são escassos. Entretanto, estudos mais aprofundados serão necessários para que as substâncias de interesse sejam elucidadas, além de testes farmacológicos e toxicológicos que forneçam embasamento científico para o uso seguro dessa espécie.

\section{REFERÊNCIAS}

AMARAL JR., A. Eritroxiláceas. In: REITZ, R.(Ed.). tajaí: Herbário "Barbosa Rodrigues", 1980. 64 p.

AMOEDO, L.H.G.; MURADIAN, L.B.A. Comparação de metodologias para determinação de umidade em geléia real. Química Nova, v. 25, n. 4, p. 676-679, 2002.

ANSEL, S.M. et al. Diterpenes from the timber of 20 Erythroxylum species. Phytochemistry, n. 32, p. 953959, 1993.

ARDISSON, L. et al. Preparação e caracterização de extratos glicólicos enriquecidos em taninos a partir das caetscas de Stryphnodendron adstringens (Mart.) Coville (Barbatimão). Revista Brasileira de Farmacognosia, v. 12, n. 1, p. 27-34, 2002.

ARRUDA, L.A. et al. Prospecção Fitoquímica, Dosagem de Fenóis, Taninos e Flavonóides Totais de Erythroxylum tortuosum Martius. 2010. 35f. Trabalho de Conclusão de Curso. Graduação em Farmácia, Centro Universitário de Anápolis - UniEvangélica.

AURICCHIO, M.T. et al. Atividades Antimicrobiana e Antioxidante e Toxicidade de Eugenia uniflora.Latin American Journal of Pharmacy v. 26, n.1, p.76-81, 2007.

BARBOSA, A.V.G; AMARAL JUNIOR, A; RIZZO, J.A. Flora dos Estados de Goiás e Tocantins: Erythroxylaceae. Coleção Rizzo, v. 29. Goiânia: Editora da UFG, 2001, p.

BARREIROS, M.L. et al. Fatty Acid Esters of Triterpenes from Erythroxylum passerinum. Jornal da Sociedade Brasileira de Química, v. 13, n. 5, p. 669-673, 2002.

BIERAS, A.C.; SAJO, M.G. Ontogenia foliar de três espécies de Erythroxylum P.Browne (Erythroxylaceae) ocorrentes no cerrado. Revista Brasileira de Botânica, n. 27, p.71-77, 2004.

BIERI, S. et al. Cocaine distribution in wild Erythroxylum species. Journal of Ethnopharmacology, v. 103, p. 439-447, 2006.

BOHM, B.A. et al. Byosystematics and evolution of cultivated coca (Erythroxylaceae).Systematic Botany, n.7, p.121-133, 1982.

BOHM, B.A. et al. Flavonoids and triterpenes from leaves of Erythroxylum nummularia. Phytochemistry, n. 27, p.833-837, 1988.

BORELLAJ.C. et al. Influência da adubação e da cobertura morta na produtividade e no teor de flavonóides de Calendula officinalis L. (Asteraceae). Revista Brasileira de Plantas Medicinais, v.13, n. 2, p. 235-239, 2011.

BRACHET, A. et al. Flavonoids and triterpenes from leaves of Erythroxylum nummularia. Phytochemistry, n. 46, p.1439- 1442, 1997.

BRASIL. Ministério da Saúde. Política nacional de plantas medicinais e fitoterápicos / Ministério da Saúde, Secretaria de Ciência, Tecnologia e Insumos Estratégicos, Departamento de Assistência Farmacêutica. Brasília, 2006. 60p.

BRASIL. Agência Nacional de Vigilância Sanitária. Farmacopéia Brasileira. 5. ed. Brasília: Anvisa, 2010. v. $1.523 p$.

BRASIL. Agência Nacional de Vigilância Sanitária. Farmacopéia Brasileira. 5. ed. Brasília: Anvisa, 2010. v. 2. 899p.

BRINGMANM, G. et al. Biochemical Systematics and Ecology: Flavonoids and triterpenes. Phytochemistry, n. 53, 2000. 409p.

BROCK, A. et al. Calystegines in wild and cultivated Erythroxylum species. Phytochemistry, v. 66, p. 12311240, 2005.

CATARINO, K.M., PAULA, J.R., DIAS, H.F.; VILA VERDE, G.M. Estudo farmacognóstico das folhas de Erythroxylum campestre A. St. -Hil. e Erythroxylum deciduum A. St. -Hil. (Erythroxylaceae). 2007. Trabalho de Conclusão de Curso, Graduação em Farmácia, Centro Universitário de Anápolis UniEvangélica. Anápolis, Goiás.

CHAVES, J.P. et al. Flavanoids and triterpene ester derivatives from Erythroxylum leal costae. Phytochemistry, v. 41, n. 3, p.941-943, 1996.

CHRISTEN, P. et al. Flavonoids and triterpenes from leaves of Erythroxylum numularia. Phytochemistry, $\mathrm{n}$. 38, p.1053-1056, 1995.

COSTA, A.C. Farmacognosia. 2 ed. Lisboa: Fundação Calouste Gulbenkian, 1982. 1032p.

COSTA, A. F. Farmacognosia. 3. ed. Lisboa: Fundação Calouste Gulbenkian, v.3, 2001

CUNHA, A.P. Farmacognosia e Fitoquímica. Lisboa: Fundação Calouste Gulbenkian, 2005. 670p.

FARIAS, M. R. Avaliação da qualidade de matériasprimas vegetais. In: SIMÕES, C. M. O. et al. (Org.). Farmacognosia: da planta ao medicamento. 5. ed. revisão. ampliada. Florianópolis: UFSC; Porto Alegre: Ed. da UFRGS, 2004. p. 263-288.

EL-IMAM, Y.M.A. et al. Flavonoids and triterpenes from leaves of Erythroxylum nummularia. Phytochemistry, v. 27, n. 7, p. 2181-2184, 1988.

EVANS, W.C. The comparative phytochemistry of the genus Erythroxylum. Journal of Ethnopharmacology, n. 3, p. 265-277, 1981.

FARMACOPÉIA BRASILEIRA. 5 ed. v.1 São Paulo: Atheneu, 2010. 523p.

FARMACOPÉIA BRASILEIRA. 5 ed. v.2 São Paulo: Atheneu, 2010. 899p.

GRIFFIN, W.J.; LIN, G.D. Chemotaxonomy and 
geographical distribuition of tropane alkaloids. Phytochemistry, n. 53, p. 623-637, 2000.

HAGERMAN, A. E.; BUTLER, L.G. Protein precipitation method for the quantitative determination of tannins. Journal of Agriculture Food Chemistry. v. 26 p. 809-812. 1978.

JOHNSON, E.L. et al. kaempferol (rhamnosyl) glucoside, a new flavonol from Erythroxylum coca. var. ipadu. Biochemical Systematics and Ecology, n. 31, p. 59-67, 2003.

KOLODZIEJ, H. et al. Flavonoids and triterpenes from leaves of Erythroxylum nummularia. Phytochemistry. n. 30, 1991. p.1255.

MATOS, F.J.A. Introdução à fitoquímica experimental. Fortaleza: UFC, 1988, 128p.

MATOS, J.M.D.; MATOS, M.E. Farmacognosia. Fortaleza: UFC, 1989, 245p.

MENDONÇA, J.O; CERVI, A.C; GUIMARÃES, O.A. O Gênero Erythroxylum P. Browne (Erythroxylaceae) do Estado do Paraná, Brasil. Brazilian Archives of Biology and Technology, v. 41 n.3, Curitiba, p.349358, 1988.

Brasil, Ministério do Meio Ambiente. 2011. Guia de campo: vegetação do Cerrado 500 espécies. Brasília: MMA/ SBF, 532p.

MOLE, S.; WATERMAN, P. G. A Critical analysis of techniques for measuring tannins in ecological studies I. Techniques for chemically defining tannins. Oecologia, v. 72, p. 137-147, 1987a

MOLE, S.; WATERMAN, P. G. A critical analysis of techniques for measuring tannins in ecological studies II. Techniques for biochemically defining tannins. Oecologia, v. 72, p. 148-156, 1987b.

NAKAMURA, A.T. Morfologia e anatomia dos frutos e sementes de três espécies de Erythroxylum P. Browne (Erythroxylaceae). 2003. Dissertação de Mestrado, Instituto de Biociências de Botucatu; Universidade Estadual Paulista.99p.

NASCIMENTO, C. J. et al. Biologically active abietane and ent-kaurane diterpenoids and other constituents from Erythroxylum suberosum. Phytochemistry Letters, $v$. 5, n. 2, p. 401-406, 2012.

OLIVEIRA, F.; AKISUE, G.; AKISUE, M. K. Farmacognosia - 1. ed. - São Paulo: Editora Atheneu, 2005.

OLIVEIRA, M. S. da Silva. et al., Tropane Alkaloids from Erythroxylum genus: distribution and compilation of ${ }^{13} \mathrm{C}-\mathrm{nmr}$ spectral data, Chemistry \& Biodiversity, v. 7, p.302-326, 2010.

OLIVEIRA, D.R; SOUZA, L.V; VILA VERDE, G.M. Doseamento de Compostos e Análise Citotóxica do Extrato Etanólico das Folhas de Erythroxylum deciduum St.-Hil. (Erythroxylaceae) pelo Bioensaio de Letalidade com Artemia Salina Leach. 2014.39f. Trabalho de Conclusão de Curso, Graduação em Farmácia, Universidade Estadual de Goias - UnUCET, Anápolis.

PAULA, P.H.S. Estudo fitoquímico da fração hexânica das folhas de Erythroxylum deciduum (Erythroxylaceae). 2012. 38p. Trabalho de Conclusão de Curso, Graduação em Química Industrial.
Universidade Estadual de Goiás - UnUCET, Anápolis.

PAYOL-HILL, A.L. et al. Flavonoids and triterpenes from leaves of Erythroxylum nummularia. Phytochemistry, n. 54, p. 927-931, 2000.

PLOWMAN, T.C.; BERRY, P.E. Erythroxylaceae. In: STEYERMARK, P. BERRY, B. HOLST e YATSKIEVYCH. Flora of the Venezuelan Guayana.St. Louis, Missouri Botanical Garden Press, n. 5, 1999. p. 59-71.

PREGNOLATTO, W; PREGNOLATTO, N.P. Normas analíticas do Instituto Adolfo Lutz. São Paulo: SP, 1985. 262p.

RIBEIRO, J.E.L.S.; HOPKINS, M.J.G.; VICENTINI, A.; SOTHERS, C.A.; COSTA, M.A.S.; BRITO,J.M.; SOUZA, M.A.D.; MARTINS, L.H.P.; LOHMANN, L.G.; ASSUNÇÃO, P.A.C.L.; PEREIRA, E.C.; SILVA, C.F.; MESQUITA, M.R. \& PROCÓPIO, L.C. Flora da Reserva Ducke: Guia de identificação das plantas vasculares de uma floresta de terra-firme na Amazônia Central. Instituto Nacional de Pesquisas da Amazônia. Manaus, 1999. 18p.

ROLIM, A. et al. Validation assay for total flavonoids, as rutin equivalents, from Trichilia catigua Adr. Juss. (Meliaceae) and Ptychopetalum olacoides Bentham (Olacaceae) commercial extract. Journal of AOAC International, v. 88, n. 4, p. 1015-1019, 2005.

ROSTIROLLA, T.M; SOUZA, K.C.B; GHILARDI, R; ANGELI, V.W. Padronização do doseamento de flavonóides totais por espectrofotometria no ultravioleta em folhas de Ginkgo biloba L. In: XVIII ENCONTRO DE JOVENS PESQUISADORES - UNIVERSIDADE DE CAXIAS DO SUL, 2010.

SANTOS, S.C.; MELLO, J.C.P. Taninos. In: SIMÕES, C.M.O. et al. Farmacognosia: da planta ao medicamento. 5. ed. Porto Alegre: Editora da Universidade Federal do Rio Grande do Sul; Florianópolis: Editora da Universidade Florianópolis, p. 615-656, 2004.

SIBIO, P. R.; Rossi, M. N., Efeito da herbívora por Evippe Sp.2 (lepidoptera:gelechiidae) na distribuição de recursos e compostos secundários em Erythroxylum tortuosum Mart. (Erythroxylaceae), Anais do VIII Congresso de Ecologia do Brasil, Caxambu - MG, 2007.

SILVA JÚNIOR, M. C. 100 árvores do cerrado: guia de campo. Brasília, Editora Rede de Sementes do Cerrado. 2005. 278p

SOUZA, V.C; LORENZI, H. Botânica sistemática: guia ilustrado para identificação das famílias de Fanerógamas nativas e exóticas no Brasil baseado em APG III.3ed. Nova Odessa: Instituto Plantarum, 2012. 768p.

ZUANAZZI, J.A.S.; MONTANHA, J.A. Flavonóides. In: SIMÕES, C.M.O. et al. Farmacognosia: da planta ao medicamento. 5. ed. Florianópolis: Editora da Universidade Federal de Santa Catarina; Porto Alegre: Editora da Universidade Federal do Rio Grande do Sul, 2004. p. 577-614.

ZUANAZZI, J.A.S. et al. Alkaloids of Erythroxylum (Erythroxylaceae) species from Southern Brazil. Biochemical Systematics and Ecology; n. 29, p. 819-825, 2001. 